\title{
AC 2007-524: MEMS FABRICATION AS A MULTIDISCIPLINARY LABORATORY
}

Todd Kaiser, Montana State University

Andrew Lingley, Montana State University

Matt Leone, Montana State University

Brad Pierson, Montana State University 


\title{
MEMS Fabrication as a Multidisciplinary Laboratory
}

\begin{abstract}
A multidisciplinary course in microelectromechanical systems (MEMS) fabrication is described in which students design a six mask process to produce accelerometers, pressure sensors and tactile sensors. The course teaches interdisciplinary tradeoffs between mechanical design, electrical design, material properties and microfabrication in a 15-week term where students fabricate, model, and test working microsystem devices. The students learn the basics of microfabrication, the impact of the fabrication process on the electrical and mechanical design of a sensor, and the effects of electrical and mechanical design on the yield of functional devices. The course is designed to run parallel to an "Introduction to Microfabrication" course where CMOS transistors are fabricated. This allows for a reduction in total resources required when compared to offering individual or separate courses.
\end{abstract}

\section{Introduction}

Microelectromechanical systems (MEMS) engineering is inherently a multidisciplinary field based on interactions of mechanical and electrical components which typically require insights into numerous other fields depending on the specific application and use of the device created. The diversity of the field overlaps the traditional boundaries between curricula. Students must have a fundamental understanding of mechanical and electrical engineering, but also need to assess the impact of fabrication, packaging and application on the design. This can require aspects of chemistry, physics, chemical engineering, material science and other assorted fields. This makes a MEMS laboratory based course an ideal channel to introduce students to multidisciplinary projects.

\section{Course Structure}

The laboratory was developed to add a MEMS portion to the current microfabrication course where CMOS transistors are fabricated and tested ${ }^{1}$. The mask set and fabrication procedures were developed as a senior design project by undergraduate students. The project is constrained in such a way that the devices are produced with the currently available cleanroom facilities used to produce CMOS transistors. This reduced tool set limits the design to bulk silicon mechanical structures and diffused piezoresistive sensing elements.

Educational materials included in the MEMS course were developed after reviewing MEMS programs at other universities ${ }^{2,3}$ and compiling information from textbooks ${ }^{10,11}$. Key to the development of this course has been frequent review and updating as the course is taught and new program descriptions ${ }^{4-9}$ and textbooks are published ${ }^{12,13}$. The lecture component of the course exists to instruct the student in the necessary information required to design, model, fabricate, and test simple MEMS structures. A review of essential electrical, mechanical, and material concepts begins in the first lectures. Electrical conductivity of materials introduces the concept of conduction bands and the classification of materials as dielectrics, semiconductors and metals. Bulk silicon structures lead to a discussion of silicon as both a mechanical and electrical material, and the crystalline nature of silicon substrate wafers. Here the ideas of stress 
and strain are introduced and lay the ground work for a discussion of flexural beam bending analysis under simple loading conditions. Next, methods for analyzing cantilever beams and suspended membranes are developed, and modeling schemes for finding modal vibrations and resonant frequencies are discussed.

The following series of lectures introduce the students to the principles of microfabrication, beginning with photolithography. Basic techniques and mask making concepts guide the discussions and additional lectures cover the limitations of the optical process. The instructor presents an analysis of the optical process. This information then proves a valuable foundation for further education regarding the concepts of variation in the manufacturing processes and statistical process control. Various deposition and etching techniques are conveyed to the student and allow an important understanding of their impact on design and ultimately the final structure. After the background material is covered, the concept of transducers is established. This begins with an introduction to the ideas of energy domains and the transfer of energy between domains. Finally, the lecture series is directed to the production of MEMS devices, typically sensors and actuators. The remainder of the term is dedicated to understanding critical issues in the complete microsystem, such as impact of the various choices in packaging, integration and partitioning of electronics, and power issues. Table 1 summarizes the content topics.

\section{Materials}

Classification

Metals

Semiconductors

Dielectrics

Conductivity

Silicon

Crystal Properties

Stress and Strain

Flexures

Cross-Discipline effects

Piezoelectric

Piezoresistive

Pyroelectric

Magnetostrictive
Fabrication

Photolithography

Etching

Wet

Dry

Deposition

Oxidation

CVD

PVD

Electroplating
Transduction

Energy Domains

Transduction

Sensors

Actuators

Techniques

Capacitive

Magnetic

Thermal

Piezoresistive

Piezoelectric
Microsystems

Circuits

Bridges

$\mathrm{A} / \mathrm{D}$

Conversion

System

Power Issues

Management

Dissipation

Noise

Sources

Limits

Packaging

Table 1. Outline of the Lecture Course Material.

\section{Fabrication}

Students use a mask set developed during the undergraduate design project to generate MEMS structures. Three undergraduate students with some microfabrication experience developed the mask set as part of their required junior level design class. Then to fulfill senior design course requirements, they confirmed the fabrication process in the MEMS laboratory. Figure 1 shows the undergraduate students discussing their poster at the engineering design fair. 


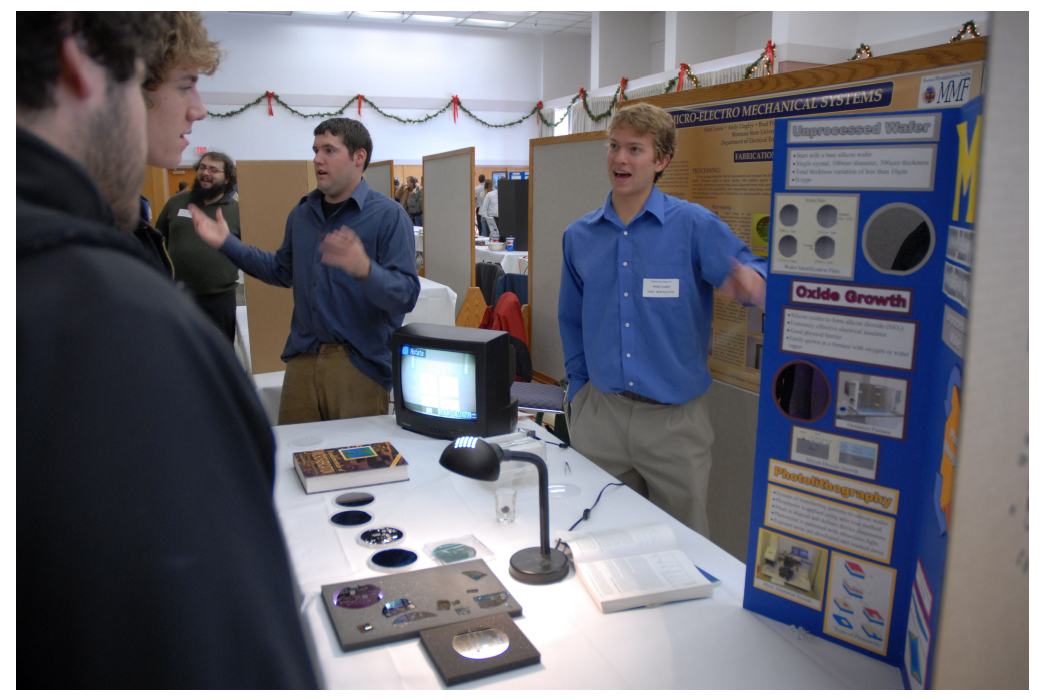

Figure 1. Engineering Design Fair Discussion

Figure 2 shows the computer aided design (CAD) layout of the devices. Numerous geometries with varying aspect ratios were incorporated into their final version. This allowed for the experimental confirmation of empirical formulas used to model the various structures. The structures included cantilever beams, cantilever beams with proof masses (accelerometers), diaphragms (pressure sensors), and suspended force sensors (tactile sensors). Also included in the design were various test structures and regions to quantify the fabrication parameters such as sheet resistance and diffusion profiles.

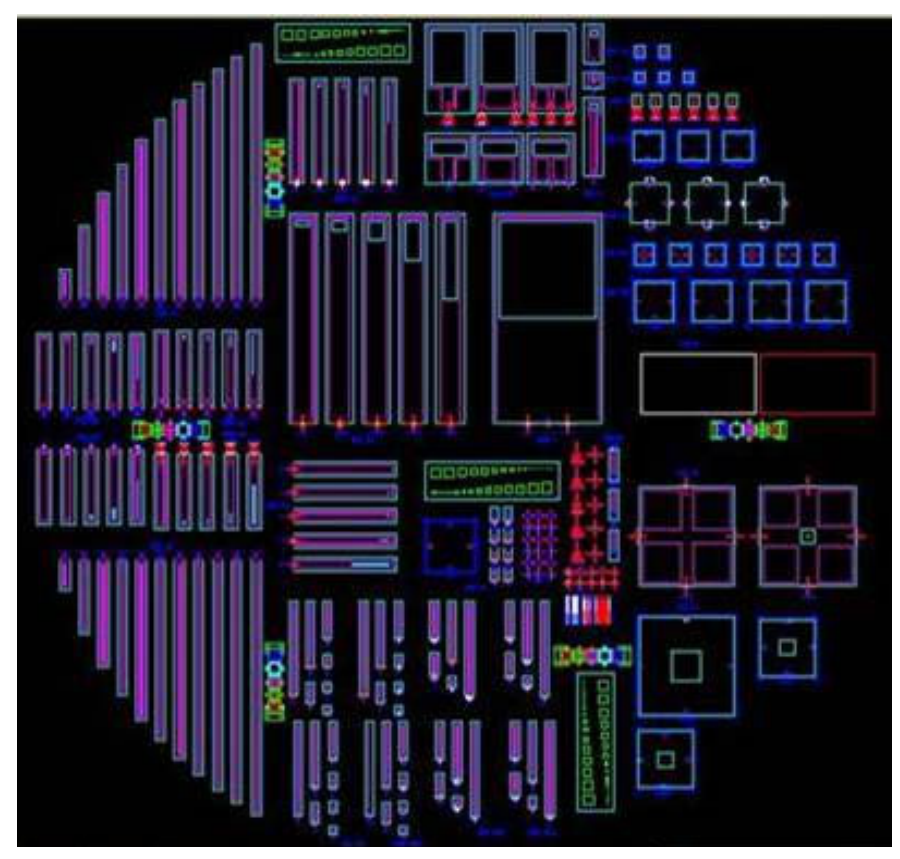

Figure 2. CAD Layout of MEMS structures, cantilever beams, accelerometers, pressure sensors and tactile sensors. 
The process flow, shown in Figure 3, starts with an n-type double side polished four inch silicon wafer (step 1). An oxide layer is grown in a wet process that typical produces 0.4 microns of oxide (step 2). The oxide is patterned and provides the masking layer for a boron diffusion that produces the piezoresistors and the p+ regions for electrical contact (steps $3 \& 4$ ). The masking oxide is then stripped in a buffered oxide etchant (step 5). A new and thicker layer of oxide is grown; this layer is approximately one micron thick. This provides the mask for the bulk etching of the silicon (step 6). A layer of aluminum is then deposited with a physical vapor deposition system. This layer of aluminum is patterned to allow for backside alignment of the bulk masking layer (steps 7-9). The silicon is then etched in a solution of Tetramethyl Ammonium Hydroxide (TMAH). This is a timed etch to produce the desired thickness of the flexures and diaphragms (step 10). The wafer has now been severely weakened and to continue processing the etched wafer is mounted on a handle wafer (step 11). Aluminum is now deposited and patterned to produce the contact to the diffused resistors (step 12). The front side oxide layer is now patterned (step 13). The final release is then done by dry etching the exposed silicon (step 14). Figure 4 shows some released structures with various designs of flexures and cantilevers.

1.

2.
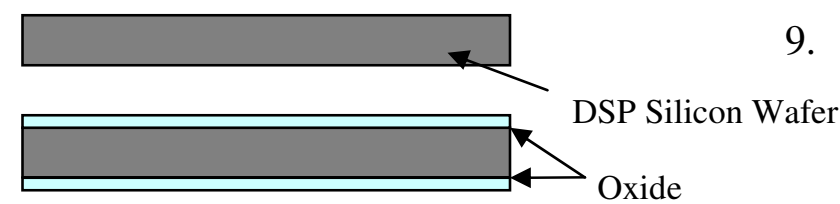

3.

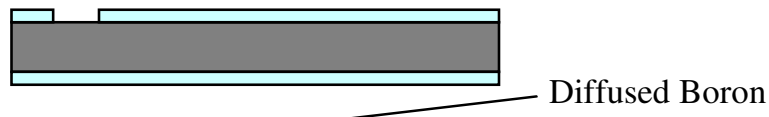

4.

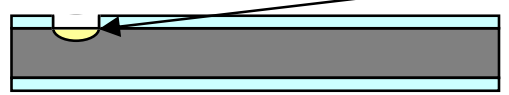

5.

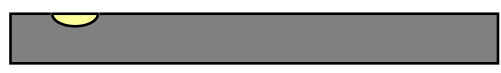

6.

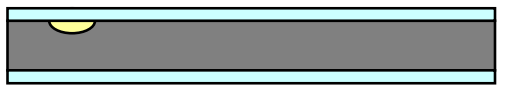

7.

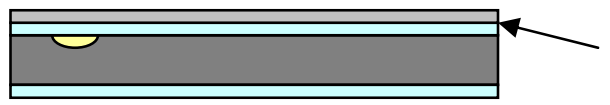

8.

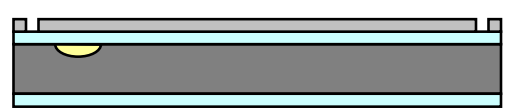

DSP Silicon Wafer

9.
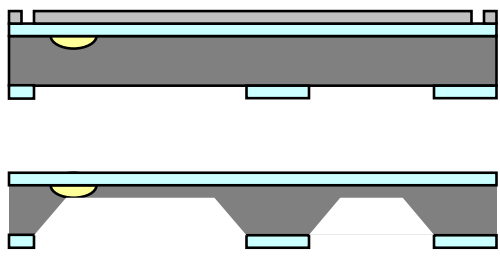

Handle Wafer

12.

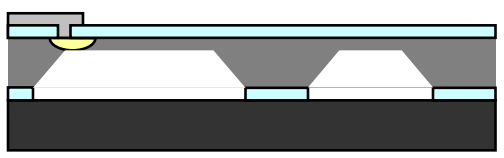

13.

Evaporated

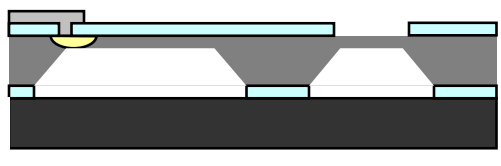

14.

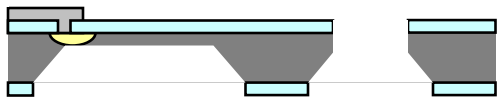

Figure 3. Basic process flow to produce MEMS structures. 


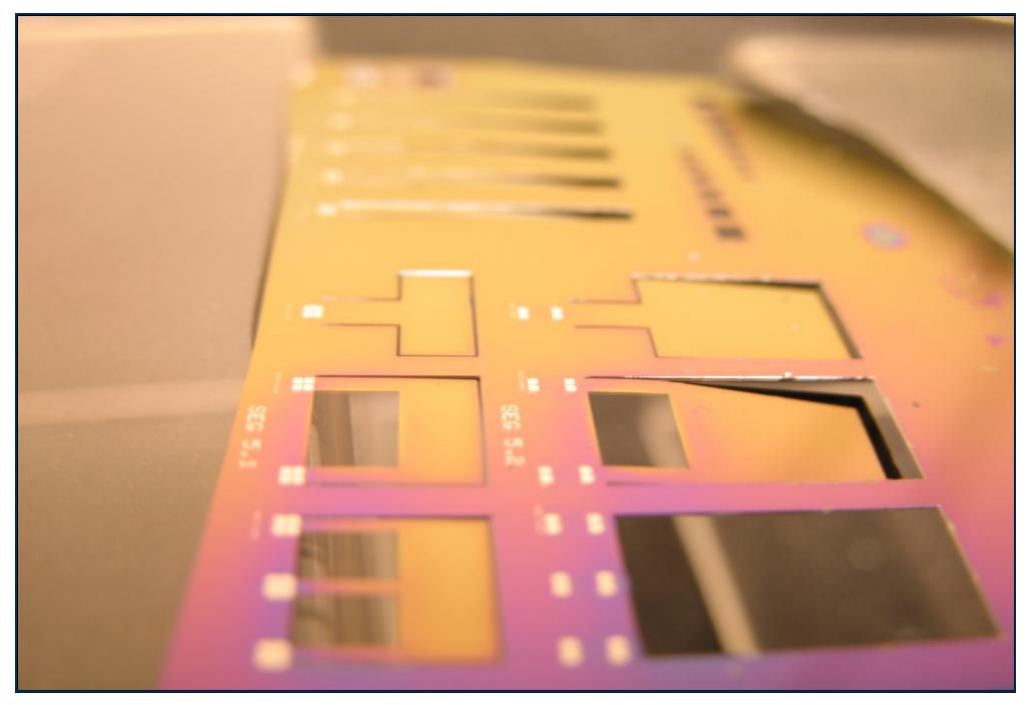

Figure 4. Released cantilever structures.

\section{Analysis and Modeling}

Prior to fabrication the students are introduced to fundamental structural principles that govern the mechanics of the cantilever devices. Following this introduction the student is assigned the task of modeling the structures that they will fabricate. Using basic beam theory, values for the effective spring constant and the mass are calculated. Next, students are informed about the equivalency between a mechanical spring-mass-dashpot system and electrical capacitor-inductor-resistor representations. Subsequently, students are then asked to model the mechanical system as an electrical system in a circuit simulation program such as P-Spice. This is completed for various damping factors so that the students see the impact that a loss element has on the quality factor of a resonant system, whether it is mechanical or electrical. The differential equations are then developed and the block-diagram for the system representation is explained. The differential equation then becomes the basis for developing a time-domain representation of the system; the input is an external force on the proof mass. When the force is divided by the mass value an expression for the acceleration is obtained. If this is integrated, the velocity of the proof mass is obtained. The damping coefficient then scales the velocity to sum into the net force acting on the proof mass. If the velocity is again integrated the position of the proof mass is obtained. The spring constant then scales the displacement to get the restoration force that is also summed into the net force acting on the proof mass. The students are then required to develop a time domain simulation of the system in MATLAB using the "Block Diagrams" in the SIMULINK toolbox. Two different representations of the system are developed, in which both models give the dynamic behavior of a mass on a spring. The final modeling of the mechanical structures is accomplished with finite element analysis to obtain the natural vibration modes of the various structures with discussions on the discrepancies between models and the impact of fabrication variations analyzed. These models will be compared to the experimental data obtained from the fabricated structures. 


\section{Model Verification}

Once the fabrication of released devices is complete, the students then compare their models to the actual devices. First, they compare the manufactured devices to the initial design of the devices. Variations in the fabrication process can produce structures that do not have the dimensions that they were designed to have. Measurements are taken to compare the actual physical sizes of the flexures and proof masses. The perturbations are then compared at different locations on the wafer to see how uniform the process is across the wafer. The new dimensions were then used to modify the models from what was designed to what was actually constructed. The dynamics of the new updated system model is compared to experimental results. The structures are placed under a probe station and contact is made to the piezoresistors. The sensing resistor is placed in a Wheatstone bridge configuration. The deformation of the piezoresistor will change its resistance and is converted to a voltage change and monitored. A measurement of the natural resonant frequency of the cantilever beam is obtained by using an additional probe tip while deflecting then releasing the cantilever beam. Figure 5 shows the results of two different cantilever beams. The experimental measurements are compared to models developed by the students. Any discrepancy between the model and experiment is analyzed to reveal the source of the discrepancy. The student adjusts the model to fit the experimental model, by changing the mass, spring constant and damping coefficient to best fit the frequency and expediential decay of the oscillation.
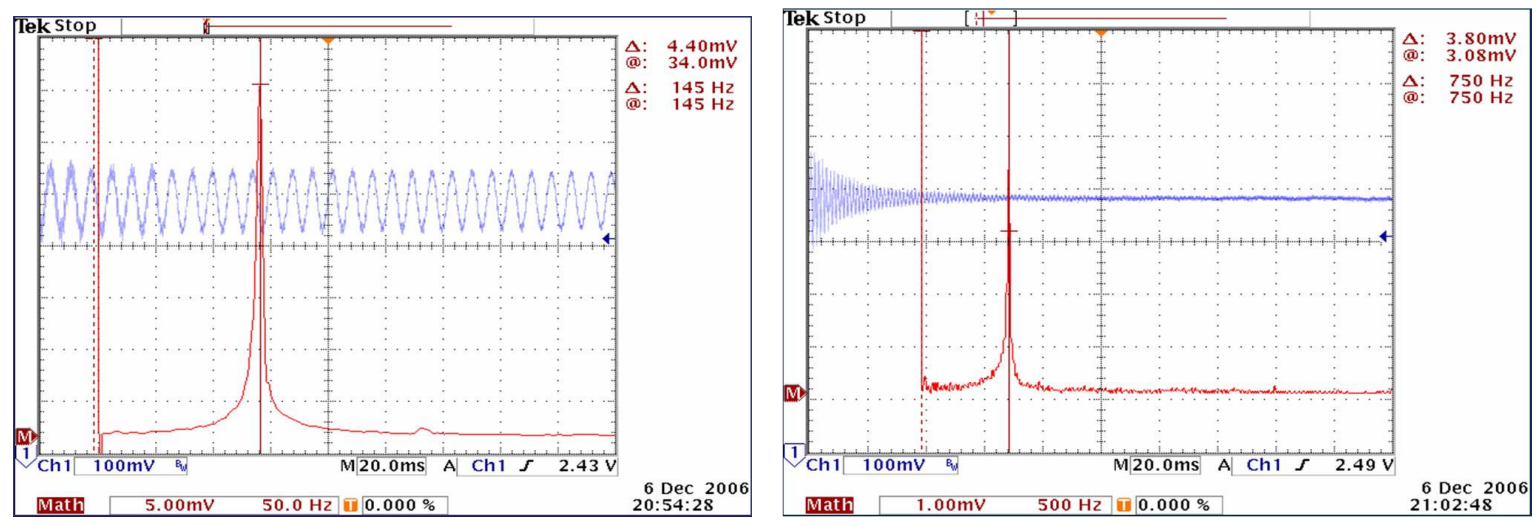

Figure 5. Typical outputs from the experimental measurements; two different structures were deflected then released to show different oscillation frequencies and damping coefficients.

\section{Conclusion}

A fifteen-week laboratory intensive course on micromachining and microsystems has been designed and focuses on the multidisciplinary aspect of the field, where elements of chemistry, physics and optics are fundamental in understanding the capabilities and limits of microfabrication. Upon completing the course, students learn to appreciate the impact that the microfabrication process has on the electrical and mechanical design of simple microstructures and how variations in the process can change both the electrical and mechanical properties of the final device. Industrial engineering concepts are introduced with consideration for which approach will produce the most robust design in terms of repeatable dimensions, material properties and performance. Furthermore, the student gains a better understanding of events that 
occur when a device goes into production, where yield and statistical quality control issues become much more important. Students learn there are trade-offs between all aspects of the design process and compromises are made due to limitations in the fabrication process, material properties, partitioning of devices and electronics, packaging, and performance. This course prepares students to be productive participants in the growing microsystems arena.

Partial support for this work was provided by the National Science Foundation's Course, Curriculum, and Laboratory Improvement program under grant 0411200.

\section{Bibliography}

1. S. Applin, T. Kaiser, "Design of a Microelectronic Manufacturing Laboratory," Proceedings of the ASEE Annual Conference, Chicago, Illinois, 2006.

2. L. Frechette, V. Modi, F. Miller, K. R. Farmer, "Hands-On MEMS," Proceedings of the $15^{\text {th }}$ Biennial Unversity/Government/Industry Microelectronics Symposium, Boise, Idaho, June 2003.

3. J. W. Judy, P. S. Motta, "A Lecture and Hands-on Laboratory Course: Introduction to Micromachining and MEMS," Proceedings of the $15^{\text {th }}$ Biennial Unversity/Government/Industry Microelectronics Symposium, Boise, Idaho, June 2003.

4. L. G. Frechette, C. W. Wong, K. Chin, G. Georgiou, K. F. Farmer, F. Miller, V. Modi, "Hands-On MEMS: Building Competence Through Practical Learning Experiences," Proceedings of the Solid-State Sensors, Actuators, and Microsystems Workshop, Hilton Head, SC, June 2006.

5. K.D. Wise, K. T. Beach, T. F. Briggs, R. J. Gordenker, M. N. Gulari, “An Interdisciplinary Laboratory Course in Microsystem Development," Proceedings of the Solid-State Sensors, Actuators, and Microsystems Workshop, Hilton Head, SC, June 2006.

6. L. C. McAffe, K. Najafi, Y. B. Gianchandani, K. D. Wise, M. M. Maharbiz, “A MEMS/Microsystem Curriculum with International Dissemination," Proceedings of the Solid-State Sensors, Actuators, and Microsystems Workshop, Hilton Head, SC, June 2006.

7. J. W. Judy, P. S. Motta, "Introductory Micromaching and MEMS Course for Graduate and Undergraduate Students," Proceedings of the Solid-State Sensors, Actuators, and Microsystems Workshop, Hilton Head, SC, June 2006.

8. A. A. Barlian, R. T. Howe, G. T. A. Kovacs, B. L. Pruitt, "Micro and Nanoscale Education at Stanford University," Proceedings of the Solid-State Sensors, Actuators, and Microsystems Workshop, Hilton Head, SC, June 2006.

9. K. Najafi, M. Maharbiz, "What Should a First College Course on MEMS be?," Proceedings of the Solid-State Sensors, Actuators, and Microsystems Workshop, Hilton Head, SC, June 2006.

10. S. D. Senturia, Microsystem Design, Kluwer Academic Publishers, 2000.

11. G. T. A. Kovacs, Micromachined Transducers Sourcebook, McGraw-Hill, 1998.

12. C. Liu, Foundations of MEMS, Pearson Education, 2006.

13. J. G. Korvink, O. Paul, MEMS: a Practical Guide to Design, Analysis and Applications, Springer, 2006. 